\title{
Primitive Integration for Content based Image Retrieval
}

\author{
Riaz Ahmed Shaikh \\ School of Computer Science \\ and Engineering, \\ UESTC, Chengdu, 611731, \\ China
}

\author{
Jian-Ping Li \\ School of Computer Science \\ and Engineering, \\ UESTC, Chengdu, 611731, \\ China
}

\author{
Asif Khan \\ School of Computer Science \\ and Engineering, \\ UESTC, Chengdu, 611731, \\ China
}

\begin{abstract}
In visual imaging and processing research area unstructured arbitrary natural scene observation and understanding is a problem. Environmental perception and object recognition is an important part of the image processing. Research approach in image processing needs to proper effective abstraction low level features, so that primitive layer integration with output of preprocess always a simultaneous phenomena for content based CBIR system. Texture, color, and shape always a considerable points for extract but need a proper algorithm and model as per image database complexity increase. Paper work approach based to proposed algorithmic model for efficient and effective retrieval.
\end{abstract}

\section{Keywords}

Feature Extraction; Neural Network; Content Based Image Retrieval; Image Analysis;

\section{INTRODUCTION}

Development and designing of an efficient and effective CBIR systems are still a challenging research problem. Two well-known problems are faced by the digital images, one is semantic gap and second is implementing efficient indexing scheme to retrieve related images from database. The semantic gap is the non similarity between high level features and low level features mainly depends on the domain knowledge to represent images. On the other hand, the computation load, when large image collections are managed, may make impractical use of CBIR systems. Proposed model based on neural network is an appropriate technique to retrieve efficient all features, from complex database. Process of feature extraction is a primary procedure to enhance the performance of image classification. Nowadays cause of security or other purpose, dynamic world screening continuously stored by different ways like CCTV cameras, but this dynamic situation create complex database that situation going to become more complex when a specific database has similar type of large images, means most of the object in every image is present in each other so this type complex situation need a new era of solution to improve the performance and efficiency of system. Proposed model provides effective and efficient solution to content based CBIR systems.

The reorganization of the particular object can be realized by using the comparison with the standard image template stored in database. After simulate the visual cortex structure to construct the object recognition is a viable approach, visual system divide the different image features (e.g., shape, motion, color, spatial position, etc.) into different layer in parallel, each of the hierarchical structure of a serial access. In all parallel processing paths, the two most important steps required from processing an image and comparative an image and then retrieve attributes towards completely access an image. "SINGLE LAYER PURIFIER INTELLIGENT
FILTER FOR IMAGE model, as per Mel and SEEMORE model or HMax model a verified approach that's reduce the complexity of layer and that performance is update and efficient.

Proposed model is an algorithmic system module by which preprocessing operations combined and functioning one by one in cyclic way to improve the image features and according to output of features image storage indexing perform in database. The low level layer that is Primitive Layer is a platform where all facts of image processing integrated to improve, to extract the features of image means here preprocessing and features extraction parallel analyzed for output and that output is also image. PURIFIER INTELLIGENT FILTER capable to integrate all preprocess and features at single platform, so query result become effective and efficient by content based image retrieval.

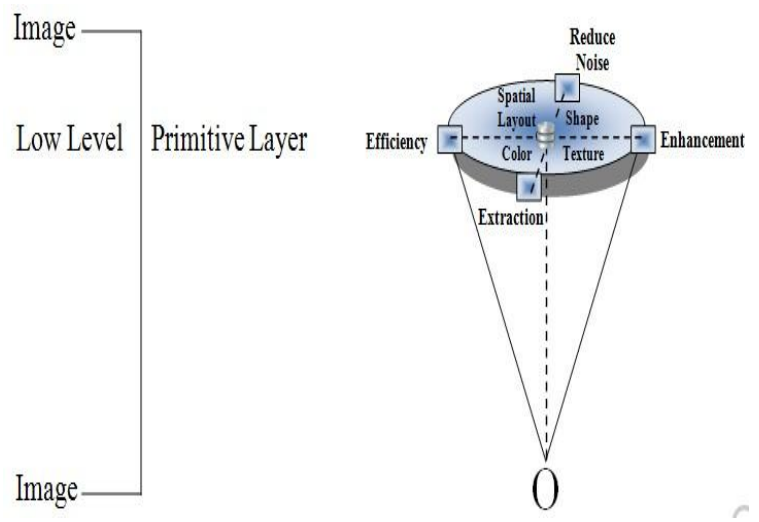

Fig. (1) : Primitive Platform Integration

Primitive layer (first layer) features of proposed model are color, shape, texture and spatial layout. Color is widely used content in content based image retrieval and recognized by human vision. Each image stored in the database is examined to calculate a color histogram that illustrates the quantity of picture element (pixels) of every color that is inside the digital image, and after this process color histogram of every digital image is stored in the image repository. In CBIR shape feature play a very crucial role. A binary image representing the extent of the object is known as shape of an object. The capability to retrieve an image by shape feature is possibly the most obvious requirement at the first level i.e. primitive level. Image is a class which has many objects, which can be acclaimed completely by their textures without any other information. There is no universal definition of texture. The ability to similarity matching based on shape can usually much helpful in differentiate among objects of digital images that are with similar color e.g. (leaves $\&$ grass, or sea $\&$ sky).

Primitive layer (first layer) processes of proposed model are enhancement, efficiency, extraction and noise reduction. The 
process of improving the quality of digital images by manipulating an image with some sort of techniques like smoothening, sharpening, or brighten an image is called image enhancement, so that results are more appropriate for further image analysis. Noise is the result of errors, can be produced during image acquisition process by the sensors, and scanner or digital camera. Noise reduction refers to remove the errors from images and make image clearer. Efficiency refers to the accuracy of digital image retrieval from the database. Convert the input image into the group of features is known as feature extraction, to extract relevant information from the digital image to fulfill the desired task.

\section{LITERATURE REVIEW}

In [1], a powerful texture extraction method Gabor filter was used, to explain the image regions' contents means universal content of image. As a universal color feature, color histogram and as a color similarity metric, histogram intersection was joined with Gabor texture, and confirmed to give about better results as that of regions based retrieval systems.

Algorithms for retrieve an image based on color histograms mostly ignore spatial layout information in the process of matching images. [2] Region matching based technique was proposed to enhance the retrieval process. This method called SCF (color and spatial feature based image retrieval). Images were compared through proposed technique and employ the spatial and color feature both, to smooth the progress of the retrieval process.

Different techniques for features extraction are discussed in [3]. In the field of content based CBIR systems various feature extraction techniques are available for visual features such as shape, spatial relationship, texture and color. For color feature, many color spaces are widely used to represent an image such as, RGB, HSV, The opponent color space and CIE Lxaxbx and CIE Lxuxvx. Color histogram, color Correlograms, color moments, coherence vector etc are representations of color feature of image. For texture feature representations are Tamura features, Co-occurrence matrices, Gabor filters, wavelet transform etc. For shape feature extraction two steps are involved, shape representation and object segmentation. Shape representation further divided into two classes which are boundary based and region based, Fourier descriptors and moment variant are most successful representations for these two classes.

In [5], needed time is too less in the comparison of color histogram, to calculate feature vectors for universal descriptors. Therefore, to improve the efficiency of CBIR system, a novel CBIR method was developed which firstly measures the global descriptor characteristics of all the images that are stored in database and then a search method based on histogram is examined only on matched databases in RGB color space. In this practice greater successful rate in the retrieval of required image was obtained.

Based on texture and color feature of image an efficient CBIR system was presented by Abuhaiba [6], to explain the image and its coherent parts means segmented regions, said system introduces three options to respond query image, either used region features, global features, or a combination of both. Gabor filter was used to explain the content of an image. As a universal color feature, color histogram and as color matching metric histogram intersection was combined with Gabor texture proved to deliver good result of retrieving images.

\section{METHODOLOGY}

The quandary involves in when an image entered as input or query image into software application and that software application is constructed to utilize CBIR techniques for obtaining visual properties, and matching them with features database. This is made to retrieve digital image from the image repository that are visually same with the query image. Features vector introduction about first layer pre-process is

$$
F(x)=\{\text { fi } \mid \text { fi }=(\text { VHSV }), \mid \leq I \leq N\}
$$

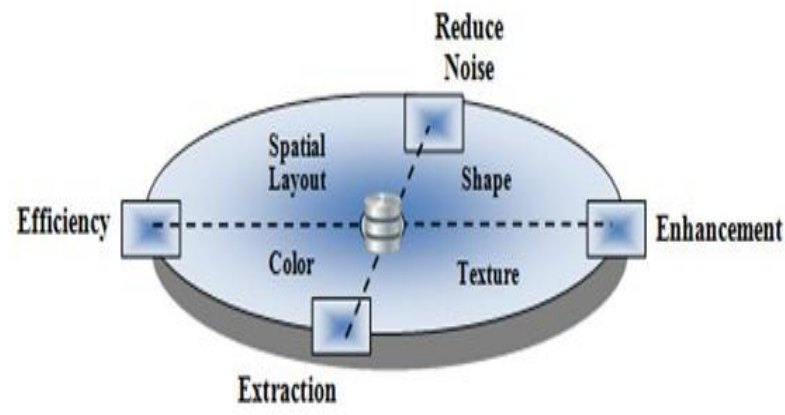

Fig. (2): Low Level Features Integration

The structure of image is much less explicit, so need to apply techniques that will identify a structure, characterizing the content of visual objects is much more complex and uncertain. Characterized by feature vectors, a visual feature is a characteristic derived by using an image analysis algorithm from transforming the original visual object.

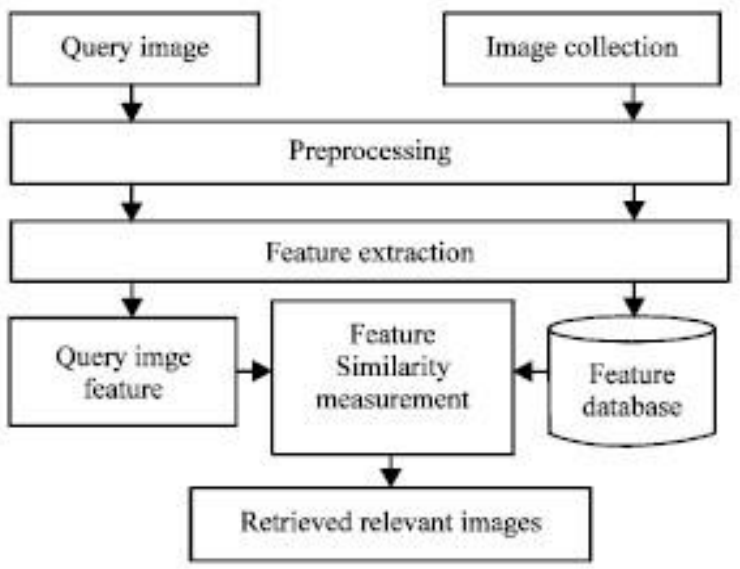

Fig. (3): Model for Primitive Integration

The query form engaged in matching the query or input image to feature of real objects which are pre-extracted. Preextracted visual features are stored in the repository, the purpose to extract a group of numerical attributes means features that eliminate redundancy from the digital image and decrease its dimensions, and color, texture and shape are most commonly used features for content based CBIR systems.

For that response output

$$
\mathbf{R}=\underset{\mathbf{j} \epsilon \mathbf{N a x}}{\operatorname{ma}} \mathbf{X}
$$

So at comparative layer as per segmentation response is depends on input. 


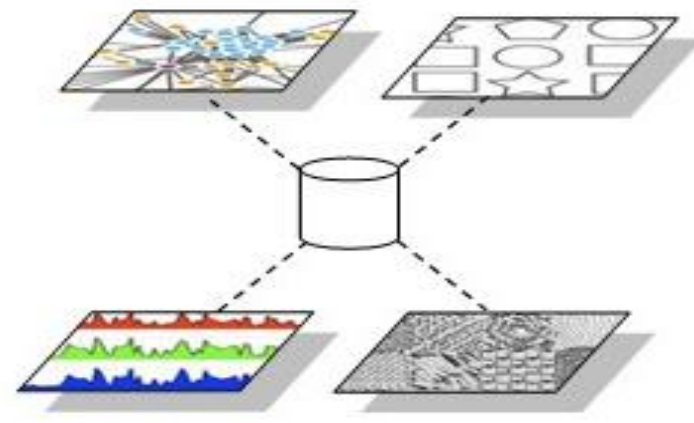

Fig. (4): Low Level Features Segmentation

Images consist of some noise and unwanted information. They should be removed from images before processing for retrieval by using filters. Different filters method can be used for removal noise. A median filter is applied on images for enhancement as a preprocessing step. Though this filter improves the image quality but it creates another problem that some amount of edge information of objects in images is lost. This edge information is recovered by applying edge extraction method. Then histogram visual features are extracted from the enhanced filtered image by quantizing histogram into bins and in each bin the average of pixels is computed which are combined to form a feature vector for retrieval of images.

Let $\mu \mathrm{j}$ is the mean and $\sigma \mathrm{j}$ is the standard deviation in a particular bin $\mathrm{j}$, where $\mathrm{j}=1,2,3 \ldots, \mathrm{L}$, and then these two features can be calculated by using the statistical measurements as:

$$
\begin{array}{r}
]_{j=}=\frac{1}{N} \sum_{i=1}^{N} X_{j i} \\
\sigma_{j}=\sqrt{\frac{1}{N} \sum_{i=1}^{N}\left(X_{j i}-\mu_{j}\right)^{2}} \\
\boldsymbol{F} \boldsymbol{V}=\{\boldsymbol{\mu} 1, \boldsymbol{\mu} 2 \ldots \boldsymbol{\mu} \boldsymbol{m}, \sigma 1, \sigma 2 \ldots \sigma \boldsymbol{m}\}
\end{array}
$$

The visual feature set is constructed and stored in the image repository for all digital images. In same way, the feature set of the input image is constructed and then contrast with the visual feature set of image repository for similarity and relevant image's retrieval.

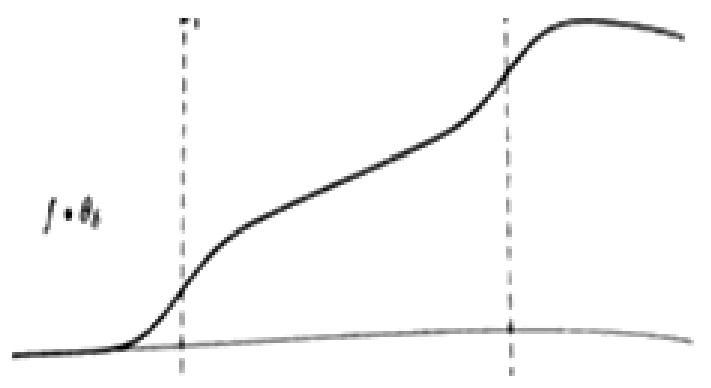

Fig. (5): Query Performance Graph
Image retrieval system based on visual contents (CBIR) system utilized visual content features of image to retrieve similar images from an image repository. CBIR systems retrieved digital images according to specified visual features stated by user. Low level features of image such as shape feature, texture feature and color feature and spatial position can reflect the visual contents of digital image.

\section{CONCLUSION}

Basic features has been introduced for retrieval of image, Since the efficiency and accuracy of texture enhanced when texture combined with shape feature and color feature, the content-based image retrieval system provides methods and techniques for querying with respect to low level visual features i.e. texture, color and shape in an integrated manner. Uses image visual content features to retrieve fast and relevant images from an image repository with low level features and preprocess, as a simultaneously filter approach provide a fast and facts result which certify as big database indexing and there object retrieval with inchoative and contemporary integration.

\section{ACKNOWLEDGEMENT}

This paper was supported by the National Natural Science Foundation of China (Grant No.61370073), the National High Technology Research and Development Program of China (Grant No.2007AA01Z423), Sichuan Province Science and technology support program (2013GZX0165), Sichuan Province Science and technology support program (2013GZ0119), Sichuan Province Science and technology support program (2014GZ0019).

\section{REFERENCES}

[1] Sunitha, S., RamaSatish, A., "Extended Image Features for User Intention Refined Image Search", International Journal of Advanced Research in Computer Science and Software Engineering, Volume 4, Issue 2, pp. 278-282, February 2014.

[2] Huu, Q., N., Thu, H., N., T., Quoc, T., N., "An Efficient Content Based Image Retreival Method for Retrieving Images", International Journal of Innovative Computing, Information and Control, Volume 8, No. 4, pp. 28232836, April 2012.

[3] Khokhar, A., Talwar, R., "Content-based Image Retrieval: Feature Extraction Techniques and Applications", International Journal of Computer Applications, (IJCS), pp. 9-14. 2012.

[4] Nascimento, M., A., Sridhar, V., Li, X., "Effective and Efficient Region-based Image Retrieval", Journal of Visual Languages and Computing, 14, pp. 151-179, 2003.

[5] Neetu Sharma. S., Paresh Rawat, S., Jaikaran Singh, S., "Efficient CBIR using Color Histogram Processing", Signal and Image Processing : An International Journal (SIPIJ) Vol. 2, No. 1, pp. 94-112, March 2011.

[6] Abuhaiba, I., S., I., Salamah, R., A., A., "Efficient Global and Region Content Based Image Retrieval", I. J. Image, Graphics and Signal Processing, 5, pp. 38-46, 2012.

[7] Rehman, M., Iqbal, M., Sharif, M., Raza, M., "Content Based Image Retrieval: Survey", World Applied Sciences Journal, 19 (3), pp. 404-412, 2012. 
[8] Amoda, N., Kulkarni, R., K., "Efficient Image Retrieval using Region Based Image Retreival", Signal \& Image Processing: An International Journal (SIPIJ) Vol. 4, No. 3, pp. 17-29, June 2013.

[9] Srinagesh, A., Aravinda, K., Saradhi Varma, G., P., Govardhan, A., SreeLatha, M., "A Modified Shape Feature Extraction Technique for Image Retrieval", International Journal of Emerging Science and Engineering (IJESE), Volume-1, Issue 8, pp. 9-13, June 2013.

[10] Chang, R., Lin, S., Y., Ho, J., M., Fann, C., W., Wang, Y., C., "A Novel Content Based Image Retrieval System Using K-Means / KNN with Feature Extraction", ComSIS Vol. 9, No. 4, Special Issue, pp. 1645-1661, December 2012.
[11] Kojic, N., S., Cabarkapa, S., K., Zajic, G., J., Reljin, B., D., "Implementation of Neural Network in CBIR Systems with Relevance Feedback", Journal of Automatic Control, University of Belgrade, Vol. 16, pp. 41-45, 2006.

[12] Yasmin, M., Sharif, M., Sajjad, M., "Use of Low Level Features for Content Based Image Retrieval: Survey", Research Journal of Recent Sciences, Vol. 2(11), pp. 6575, November 2013.

[13] Santhosh, D., Trueman, T., E., "Artificial Neural Network Technique for CBIR Based on Query Image Feature Extraction", International Journal of Innovative Research in Computer, Vol. 2, Special Issue 3, pp. 223 230, July 2014. 